\title{
CONVOLUTION ROOTS AND DIFFERENTIABILITY OF ISOTROPIC POSITIVE DEFINITE FUNCTIONS ON SPHERES
}

\author{
JOHANNA ZIEGEL
}

(Communicated by Ken Ono)

\begin{abstract}
We prove that any isotropic positive definite function on the sphere can be written as the spherical self-convolution of an isotropic real-valued function. It is known that isotropic positive definite functions on $d$-dimensional Euclidean space admit a continuous derivative of order $[(d-1) / 2]$. We show that the same holds true for isotropic positive definite functions on spheres and prove that this result is optimal for all odd dimensions.
\end{abstract}

\section{INTRODUCTION}

For an integer $d \in \mathbb{N}$ we denote the $d$-dimensional unit sphere by $\mathbb{S}^{d}=\{x \in$ $\left.\mathbb{R}^{d+1} \mid\|x\|=1\right\}$, where $\|\cdot\|$ denotes the Euclidean norm on $\mathbb{R}^{d+1}$. A function $f: \mathbb{S}^{d} \times \mathbb{S}^{d} \rightarrow \mathbb{R}$ is positive definite if

$$
\sum_{i=1}^{n} \sum_{j=1}^{n} c_{i} c_{j} f\left(u_{i}, u_{j}\right) \geq 0
$$

for all $u_{1}, \ldots, u_{n} \in \mathbb{S}^{d}$ and coefficients $c_{1}, \ldots, c_{n} \in \mathbb{R}$. The function $f$ is isotropic if there exists a function $\bar{f}:[0, \pi] \rightarrow \mathbb{R}$ that fulfills

$$
f(u, v)=\bar{f}(\theta(u, v)) \quad \text { for all } u, v \in \mathbb{S}^{d},
$$

where the geodesic distance on $\mathbb{S}^{d}$ is given by $\theta: \mathbb{S}^{d} \times \mathbb{S}^{d} \rightarrow \mathbb{R}, \theta(u, v)=\arccos (\langle u, v\rangle)$. Here, $\langle\cdot, \cdot\rangle$ denotes the standard scalar product on $\mathbb{R}^{d+1}$.

Isotropic positive definite functions on spheres occur in statistics as correlation functions of homogeneous random fields on spheres or of star-shaped random particles. They also have applications in approximation theory where they are used as radial basis functions for interpolating scattered data on spherical domains. Recent applications in spatial statistics can be found in 1, 12,13; application examples in approximation theory are given in [4,9,26].

The class $\Psi_{d}$ consists of all continuous functions $\psi:[0, \pi] \rightarrow \mathbb{R}$ with $\psi(0)=1$, such that the isotropic function $\psi(\theta(\cdot, \cdot))$ is positive definite. The classes $\Psi_{d}$ are nonincreasing in $d$,

$$
\Psi_{1} \supset \Psi_{2} \supset \cdots \supset \Psi_{\infty}=\bigcap_{d=1}^{\infty} \Psi_{d},
$$

with the inclusions being strict; see [11, Corollary 1].

Received by the editors July 4, 2012.

2010 Mathematics Subject Classification. Primary 42A82, 33C50, 33C55, 60E10.

Key words and phrases. Convolution root, covariance function, isotropic positive definite function, radial basis function, spherical convolution, turning bands operator. 
We define the spherical convolution of two isotropic functions $f, g: \mathbb{S}^{d} \times \mathbb{S}^{d} \rightarrow \mathbb{R}$ as

$$
(f \circledast g)(u, v)=\int_{\mathbb{S}^{d}} \bar{f}(\theta(u, w)) \bar{g}(\theta(w, v)) d w, \quad \text { for all } u, v \in \mathbb{S}^{d},
$$

where the integration is with respect to the $d$-dimensional Hausdorff measure on $\mathbb{S}^{d}$. The total measure of $\mathbb{S}^{d}$ is denoted by $\sigma_{d}=2 \pi^{(d+1) / 2} / \Gamma((d+1) / 2)$. It is easy to see that the spherical self-convolution of any isotropic $L^{2}$-function $f$ on $\mathbb{S}^{d} \times \mathbb{S}^{d}$ is positive definite. A function $\varphi:[0, \pi] \rightarrow \mathbb{R}$ has a spherical convolution root if there exists an isotropic function $g: \mathbb{S}^{d} \times \mathbb{S}^{d} \rightarrow \mathbb{R}$ such that $\varphi(\theta(\cdot, \cdot))=g \circledast g$.

Spherical convolution has been used by several authors [8, 12, 21, 25] as a tool to construct spherical positive definite functions. It is natural to ask the reverse question: Which functions can be obtained through this construction principle? We can give the following general positive answer, which we prove in Section 3 .

Theorem 1.1. Any $\psi \in \Psi_{d}$ has a spherical convolution root which can be taken to be real-valued and isotropic.

The techniques used to show the convolution representation theorem have led to the solution of a further interesting problem concerning positive definite functions on spheres.

A positive definite function $f$ on $\mathbb{R}^{d}$ is defined analogously to (1.1). The function $f$ is called radial if $f(x, y)=\tilde{f}(\|x-y\|)$ for some function $\tilde{f}:[0, \infty) \rightarrow \mathbb{R}$. Schoenberg [19, Lemma 4] showed that radial positive definite functions on $\mathbb{R}^{d}$ have a continuous derivative of order $[(d-1) / 2]$, where $[c]$ denotes the greatest integer less than or equal to $c$. The following theorem, which will be shown in Section 4.1 confirms the conjecture of Gneiting [11] that the same holds true on spheres.

Theorem 1.2. The functions in the class $\Psi_{d}$ admit a continuous derivative of order $[(d-1) / 2]$ on the open interval $(0, \pi)$.

The derivatives at the point $\vartheta=0$ can be infinite or can take finite values. We believe that the same holds true at $\vartheta=\pi$. However, we are currently not able to provide simple examples for the latter claim. The powered exponential family

$$
\psi(\vartheta)=\exp \left(-\left(\frac{\vartheta}{c}\right)^{\alpha}\right), \quad \vartheta \in[0, \pi]
$$

with parameters $c>0$ and $\alpha \in(0,1]$ belongs to $\Psi_{\infty}$ [11. For $\alpha<1$ the first derivative at zero is $-\infty$, whereas for $\alpha=1$ it takes the value $-1 / c$. The sine power function

$$
\psi(\vartheta)=1-\left(\sin \frac{\vartheta}{2}\right)^{\alpha}, \quad \vartheta \in[0, \pi]
$$

as in [22] is a member of $\Psi_{\infty}$ for $\alpha \in[0,2]$. For $\alpha \in(0,1)$, the first derivative at zero is $-\infty$; for $\alpha=1$, we obtain $\psi^{\prime}(0)=-1 / 2$. If $\alpha \in(1,2]$, the derivative at zero is zero.

In the Euclidean case it is known that Theorem 1.2 is the best possible [10]. Hence, there are radial positive definite functions on $\mathbb{R}^{d}$ whose derivative of order $[(d-1) / 2]+1$ is not continuous. The optimality of Theorem 1.2 for $d=1,3,5,7$ follows from the results in [2]. In Section 4.2 we introduce a turning bands operator for isotropic positive definite functions on spheres to show the optimality of Theorem 1.2 for all odd dimensions. In even dimensions it remains an open problem. However, once the optimality can be shown for $d=2$, the turning bands operator immediately yields the assertion in all even dimensions as well. 
The convolution representation result, Theorem 1.1, also has consequences that are of interest in statistical applications. Firstly, it shows that any isotropic covariance function on the sphere can be obtained by the Lévy based approach to modelling star-shaped random particles introduced in 12. Secondly, the proof of Theorem 1.1 reveals a way to resolve the identifiability issues associated with these models. It is possible to distinguish one specific convolution root amongst all possible convolution roots of a given covariance function. This is the basis of the inference procedure described in [27.

\section{Convolution of isotropic Functions on SPheres}

Let $L^{2}\left(\mathbb{S}^{d} \times \mathbb{S}^{d}\right)$ be the space of square-integrable functions on $\mathbb{S}^{d} \times \mathbb{S}^{d}$ with the Hausdorff measure. By $\langle\cdot, \cdot\rangle_{L^{2}}$ and $\|\cdot\|_{L^{2}}$ we denote the scalar product and the norm of the Hilbert space $L^{2}\left(\mathbb{S}^{d} \times \mathbb{S}^{d}\right)$, respectively. We consider the subspace $L_{d, \mathcal{I}}^{2} \subset L^{2}\left(\mathbb{S}^{d} \times \mathbb{S}^{d}\right)$ of functions that are isotropic as defined at (1.2). For $f \in L_{d, \mathcal{I}}^{2}$ it holds for all $d+1$-dimensional orthogonal matrices $R$ that

$$
f(R u, R v)=\bar{f}(\theta(R u, R v))=\bar{f}(\theta(u, v))=f(u, v), \quad u, v \in \mathbb{S}^{d} .
$$

This property characterizes the functions in $L_{d, \mathcal{I}}^{2}$.

Proposition 2.1. The convolution $f \circledast g$ of $f, g \in L_{d, \mathcal{I}}^{2}$ is in $L_{d, \mathcal{I}}^{2}$ and

$$
\|f \circledast g\|_{L^{2}} \leq \sigma_{d} \sup _{u, v \in \mathbb{S}^{d}}|(f \circledast g)(u, v)| \leq\|f\|_{L^{2}}\|g\|_{L^{2}} .
$$

The convolution is bilinear, commutative and

$$
\|f \circledast g\|_{L^{2}}^{2}=\langle f \circledast f, g \circledast g\rangle_{L^{2}} .
$$

Proof. It is easy to check that $f \circledast g$ is isotropic. Furthermore, by Hölder's inequality,

$$
\begin{aligned}
|(f \circledast g)(u, v)| & \leq \int_{\mathbb{S}^{d}}|\bar{f}(\theta(u, w)) \bar{g}(\theta(w, v))| d w \\
& \leq\left\{\int_{\mathbb{S}^{d}} \bar{f}(\theta(u, w))^{2} d w\right\}^{\frac{1}{2}}\left\{\int_{\mathbb{S}^{d}} \bar{g}(\theta(w, v))^{2} d w\right\}^{\frac{1}{2}} \\
& \stackrel{(*)}{=}\left\{\frac{1}{\sigma_{d}} \int_{\mathbb{S}^{d} \times \mathbb{S}^{d}} \bar{f}(\theta(u, w))^{2} d w d u\right\}^{\frac{1}{2}}\left\{\frac{1}{\sigma_{d}} \int_{\mathbb{S}^{d} \times \mathbb{S}^{d}} \bar{g}(\theta(w, v))^{2} d w d v\right\}^{\frac{1}{2}} \\
& =\frac{1}{\sigma_{d}}\|f\|_{L^{2}}\|g\|_{L^{2}}
\end{aligned}
$$

for $u, v \in \mathbb{S}^{d}$. The equality at $(*)$ holds true because the integrals on the left hand side do not depend on $u, v$, respectively. Therefore, we obtain (2.1), and, in particular, $f \circledast g \in L^{2}\left(\mathbb{S}^{d} \times \mathbb{S}^{d}\right)$. Bilinearity and commutativity are clear, and equation (2.2) is an application of Fubini's theorem.

Schoenberg [20] characterized the functions of the classes $\Psi_{d}$ using Gegenbauer (or ultraspherical) polynomials. Let $\lambda>0$. The Gegenbauer polynomials $C_{n}^{\lambda}$ for $n \in \mathbb{N}_{0}$ are defined by the expansion

$$
\frac{1}{\left(1+r^{2}-2 r \cos \vartheta\right)^{\lambda}}=\sum_{n=0}^{\infty} r^{n} C_{n}^{\lambda}(\cos \vartheta), \quad \text { for } \vartheta \in[0, \pi]
$$


see [6, 18.12.4]. Note that we use $\mathbb{N}$ to denote the positive integers and $\mathbb{N}_{0}=\mathbb{N} \cup\{0\}$. We will repeatedly use the fact that

$$
C_{n}^{\lambda}(1)=\frac{\Gamma(n+2 \lambda)}{n ! \Gamma(2 \lambda)} .
$$

If $\lambda=0$ we set $C_{n}^{0}(\cos \vartheta)=\cos (n \vartheta)$ for $\vartheta \in[0, \pi]$ as in $[20$. We need the following important property of the Gegenbauer polynomials with $\lambda=(d-1) / 2$; see for example [18, Corollary 4.9]. For $d \geq 2, k, n \in \mathbb{N}_{0}$ and $u, v \in \mathbb{S}^{d}$, we have

$$
\int_{\mathbb{S}^{d}} C_{k}^{(d-1) / 2}(\langle u, w\rangle) C_{n}^{(d-1) / 2}(\langle w, v\rangle) d w=\delta_{k, n} \sigma_{d} \frac{d-1}{2 n+d-1} C_{n}^{(d-1) / 2}(\langle u, v\rangle),
$$

where $\delta_{k, n}$ denotes the Kronecker delta. If $\lambda=0$ it holds that

$$
\int_{\mathbb{S}^{1}} C_{k}^{0}(\langle u, w\rangle) C_{n}^{0}(\langle w, v\rangle) d w=\delta_{k, n} \pi C_{n}^{0}(\langle u, v\rangle)
$$

for $n \in \mathbb{N}_{0}, k \in \mathbb{N}, u, v \in \mathbb{S}^{d}$, and $\int_{\mathbb{S}^{1}} C_{0}^{0}(\langle u, w\rangle) C_{0}^{0}(\langle w, v\rangle) d w=2 \pi$.

Proposition 2.2. Let $d \geq 2$. The family $\mathcal{C}_{d}=\left\{E_{d, n}\right\}_{n \in \mathbb{N}_{0}}$, where $E_{d, n}:=$ $c_{d, n} C_{n}^{(d-1) / 2}(\langle\cdot, \cdot\rangle) \in L_{d, \mathcal{I}}^{2}$ with

$$
c_{d, n}=\sigma_{d}^{-1} \sqrt{\frac{2 n+d-1}{(d-1) C_{n}^{(d-1) / 2}(1)}},
$$

is an orthonormal basis of $L_{d, \mathcal{I}}^{2}$. Furthermore, for $k, n \in \mathbb{N}_{0}$,

$$
E_{d, k} \circledast E_{d, n}=\delta_{k, n} \bar{c}_{d, n} E_{d, n},
$$

where

$$
\bar{c}_{d, n}=\sqrt{\frac{d-1}{(2 n+d-1) C_{n}^{(d-1) / 2}(1)}} .
$$

Proof. By (2.4)

$$
\begin{aligned}
\int_{\mathbb{S}^{d} \times \mathbb{S}^{d}} C_{k}^{(d-1) / 2}(\langle u, v\rangle) & C_{n}^{(d-1) / 2}(\langle u, v\rangle) d u d v \\
& =\delta_{k, n} \sigma_{d} \frac{d-1}{2 n+d-1} \int_{\mathbb{S}^{d}} C_{n}^{(d-1) / 2}(\langle v, v\rangle) d v \\
& =\delta_{k, n} \sigma_{d}^{2} \frac{d-1}{2 n+d-1} C_{n}^{(d-1) / 2}(1) ;
\end{aligned}
$$

hence $\mathcal{C}_{d}$ is an orthonormal system. It is also a Hilbert space basis, because polynomials are dense in $L^{2}([-1,1])$. The second assertion is a direct consequence of (2.4).

The following proposition complements Proposition 2.2 and is not hard to prove.

Proposition 2.3. Proposition 2.2 also holds for $d=1$ with

$$
c_{1, n}=\left\{\begin{array}{ll}
1 /(2 \pi), & \text { for } n=0, \\
\sqrt{2} /(2 \pi), & \text { for } n \geq 1,
\end{array}, \quad \bar{c}_{1, n}= \begin{cases}1, & \text { for } n=0, \\
\sqrt{2} / 2, & \text { for } n \geq 1 .\end{cases}\right.
$$


Propositions 2.2 and 2.3 imply that, for any function $f \in L_{d, \mathcal{I}}^{2}$, we have

$$
f \stackrel{L^{2}}{=} \sum_{n \in \mathbb{N}_{0}}\left\langle f, E_{d, n}\right\rangle_{L^{2}} E_{d, n}
$$

where $\stackrel{L^{2}}{=}$ means that the series on the right hand side converges unconditionally in $L^{2}$ to the left hand side. We call the basis $\mathcal{C}_{d}$ the Gegenbauer basis of $L_{d, \mathcal{I}}^{2}$. The coefficients $\left\langle f, E_{d, n}\right\rangle_{L^{2}}$ are termed the Gegenbauer coefficients of $f$.

Proposition 2.4. For any $f \in L_{d, \mathcal{I}}^{2}, n \in \mathbb{N}_{0}$, we have

$$
f \circledast E_{d, n}=\bar{c}_{d, n}\left\langle f, E_{d, n}\right\rangle_{L^{2}} E_{d, n} .
$$

Proof. For $N \in \mathbb{N}$ we set $f_{N}=\sum_{k=0}^{N}\left\langle f, E_{d, k}\right\rangle_{L^{2}} E_{d, k}$. Then $f_{N}$ converges to $f$ in $L^{2}$. We obtain

$$
\begin{aligned}
& \left\|f \circledast E_{d, n}-\bar{c}_{d, n}\left\langle f, E_{d, n}\right\rangle_{L^{2}} E_{d, n}\right\|_{L^{2}} \\
& \quad \leq\left\|f \circledast E_{d, n}-f_{N} \circledast E_{d, n}\right\|_{L^{2}}+\left\|f_{N} \circledast E_{d, n}-\bar{c}_{d, n}\left\langle f, E_{d, n}\right\rangle_{L^{2}} E_{d, n}\right\|_{L^{2}} .
\end{aligned}
$$

The last summand on the right hand side is zero by the definition of $f_{N}$ and Proposition 2.2. By Proposition 2.1 we obtain

$$
\left\|f \circledast E_{d, n}-f_{N} \circledast E_{d, n}\right\|_{L^{2}}=\left\|\left(f-f_{N}\right) \circledast E_{d, n}\right\|_{L^{2}} \leq\left\|f-f_{N}\right\|_{L^{2}}\left\|E_{d, n}\right\|_{L^{2}} \rightarrow 0,
$$

as $N \rightarrow \infty$.

Corollary 2.5. For any $f \in L_{d, \mathcal{I}}^{2}, n \in \mathbb{N}_{0}$ we have

$$
\left\langle f \circledast f, E_{d, n}\right\rangle_{L^{2}}=\bar{c}_{d, n}\left\langle f, E_{d, n}\right\rangle_{L^{2}}^{2} .
$$

Proof. We have

$$
\begin{aligned}
\left\langle f \circledast f, E_{d, n}\right\rangle_{L^{2}} & =\left(\bar{c}_{d, n}\right)^{-1}\left\langle f \circledast f, E_{d, n} \circledast E_{d, n}\right\rangle_{L^{2}}=\left(\bar{c}_{d, n}\right)^{-1}\left\|f \circledast E_{d, n}\right\|_{L^{2}}^{2} \\
& =\left(\bar{c}_{d, n}\right)^{-1}\left\|\bar{c}_{d, n}\left\langle f, E_{d, n}\right\rangle_{L^{2}} E_{d, n}\right\|_{L^{2}}^{2}=\bar{c}_{d, n}\left\langle f, E_{d, n}\right\rangle_{L^{2}}^{2},
\end{aligned}
$$

where we used Propositions 2.2 and 2.3 , equation (2.2), and Proposition 2.4 in this order.

The following theorem gives a necessary condition for the existence of convolution roots in $L_{d, \mathcal{I}}^{2}$. In the interesting special case of nonnegative Gegenbauer coefficients this condition is also sufficient.

Theorem 2.6. If a function $f \in L_{d, \mathcal{I}}^{2}$ can be represented as $f=g \circledast g$ for some $g \in L_{d, \mathcal{I}}^{2}$, then

$$
\sum_{n=0}^{\infty}\left(\bar{c}_{d, n}\right)^{-1}\left|\left\langle f, E_{d, n}\right\rangle_{L^{2}}\right|<\infty .
$$

If (2.5) holds and $\left\langle f, E_{d, n}\right\rangle_{L^{2}} \geq 0$ for all $n \in \mathbb{N}_{0}$, then there exists a $g \in L_{d, \mathcal{I}}^{2}$ such that $f=g \circledast g$. The coefficients of $g$ in the Gegenbauer basis can be chosen to be nonnegative.

Proof. The Hilbert space $L_{d, \mathcal{I}}^{2}$ is isometric to the space $\ell^{2}$ [24, Corollary V.4.13]. Therefore $\sum_{n \in \mathbb{N}_{0}} a_{n} E_{d, n} \in L_{d, \mathcal{I}}^{2}$ if and only if $\left(a_{n}\right)_{n \in \mathbb{N}_{0}} \in \ell^{2}$ or, equivalently, 
$\sum_{n=0}^{\infty} a_{n}^{2}<\infty$. Suppose now that $f$ is given by $f=g \circledast g$ for some $g \in L_{d, \mathcal{I}}^{2}$. By Corollary 2.5 we have that

$$
\left\langle g, E_{d, n}\right\rangle_{L^{2}}= \pm\left(\bar{c}_{d, n}\right)^{-\frac{1}{2}}\left|\left\langle f, E_{d, n}\right\rangle_{L^{2}}\right|^{\frac{1}{2}}
$$

hence

$$
\sum_{n=0}^{\infty}\left(\bar{c}_{d, n}\right)^{-1}\left|\left\langle f, E_{d, n}\right\rangle_{L^{2}}\right|<\infty .
$$

For the reverse implication set $g=\sum_{n \in \mathbb{N}_{0}}\left(\bar{c}_{d, n}\right)^{-1 / 2}\left\langle f, E_{d, n}\right\rangle_{L^{2}}^{1 / 2} E_{d, n}$. By assumption $g \in L_{d, \mathcal{I}}^{2}$ and by Corollary 2.5 we have for any $n \in \mathbb{N}_{0}$ that

$$
\left\langle g \circledast g, E_{d, n}\right\rangle_{L^{2}}=\bar{c}_{d, n}\left\langle g, E_{d, n}\right\rangle_{L^{2}}^{2}=\left\langle f, E_{d, n}\right\rangle_{L^{2}} .
$$

With Parseval's equality [24, Theorem V.4.9] this yields the claim.

We conclude this section with a proposition that shows that convolution products can be uniformly approximated with respect to the Gegenbauer basis $\mathcal{C}_{d}$.

Proposition 2.7. If $f \in L_{d, \mathcal{I}}^{2}$ is given by $f=g \circledast g$ for some $g \in L_{d, \mathcal{I}}^{2}$, then for every permutation $\sigma: \mathbb{N} \rightarrow \mathbb{N}$, the sequence $\left(f_{N}\right)_{N \in \mathbb{N}}$ with $f_{N}=\sum_{k=0}^{N}\left\langle f, E_{d, \sigma(k)}\right\rangle_{L^{2}} E_{d, \sigma(k)}$ converges uniformly to $f$.

Proof. Let $g_{N}=\sum_{k=0}^{N}\left\langle g, E_{d, \sigma(k)}\right\rangle_{L^{2}} E_{d, \sigma(k)}$. By Corollary 2.5 and Proposition 2.4 we have

$$
\begin{aligned}
f-f_{N} & =g \circledast g-\sum_{k=0}^{N} \bar{c}_{d, \sigma(k)}\left\langle g, E_{d, \sigma(k)}\right\rangle_{L^{2}}^{2} E_{d, \sigma(k)} \\
& =g \circledast g-\sum_{k=0}^{N}\left\langle g, E_{d, \sigma(k)}\right\rangle_{L^{2}} g \circledast E_{d, \sigma(k)}=g \circledast g-g \circledast g_{N}=g \circledast\left(g-g_{N}\right) .
\end{aligned}
$$

Now, we can apply Proposition 2.1 to the last term and use the unconditional $L^{2}$-convergence of $g_{N}$ to $g$ in order to obtain the claim.

\section{Convolution roots}

Schoenberg's characterization of the classes $\Psi_{d}$ is summarized in the following theorem; cf. [20].

Theorem 3.1 (Schoenberg). The class $\Psi_{d}$ consists of all functions of the form

$$
\psi(\vartheta)=\sum_{n=0}^{\infty} b_{d, n} \frac{C_{n}^{(d-1) / 2}(\cos \vartheta)}{C_{n}^{(d-1) / 2}(1)}, \quad \text { for } \vartheta \in[0, \pi],
$$

with nonnegative coefficients $b_{d, n}$, such that $\sum_{n=0}^{\infty} b_{d, n}=1$. If $d=1$, then

$$
b_{1,0}=\frac{1}{\pi} \int_{0}^{\pi} \psi(\vartheta) d \vartheta \quad \text { and } \quad b_{1, n}=\frac{2}{\pi} \int_{0}^{\pi} \cos (n \vartheta) \psi(\vartheta) d \vartheta, \quad \text { for } n \geq 1 .
$$

If $d \geq 2$, then, for $n \in \mathbb{N}_{0}$,

$$
b_{d, n}=\frac{2 n+d-1}{2^{3-d} \pi} \frac{\left(\Gamma\left(\frac{d-1}{2}\right)\right)^{2}}{\Gamma(d-1)} \int_{0}^{\pi}\left\{C_{n}^{(d-1) / 2}(\cos \vartheta)\right\}(\sin \vartheta)^{d-1} \psi(\vartheta) d \vartheta
$$


For a function $\psi \in \Psi_{d}$, we call the associated coefficients $b_{d, n}$ as given by (3.1) or (3.2), respectively, the d-dimensional Schoenberg coefficients of $\psi$.

A function $\psi \in \Psi_{d}$ is strictly positive definite if the inequality in (1.1) is strict for all systems of pairwise distinct points, unless all the coefficients are zero. A function $\psi \in \Psi_{d}$ for $d \geq 2$ is strictly positive definite if and only if its Schoenberg coefficients $b_{d, n}$ are strictly positive for infinitely many even and infinitely many odd integers $n$ [5]. The corresponding result for $\Psi_{\infty}$ was derived in [15]. Characterizations of the strictly positive definite functions in $\Psi_{1}$ in terms of nonzero Schoenberg coefficients are available in [16,17].

We prove the following result, which is slightly more detailed than Theorem 1.1 .

Theorem 3.2. For any $\psi \in \Psi_{d}$ there exists a function $g \in L_{d, \mathcal{I}}^{2}$ such that

$$
\psi(\theta(u, v))=(g \circledast g)(u, v), \quad \text { for all } u, v \in \mathbb{S}^{d},
$$

and $g$ has nonnegative Gegenbauer coefficients.

Proof. First, let $d \geq 2, \psi \in \Psi_{d}$. The nonnegative Schoenberg coefficients of $\psi$ are connected to the Gegenbauer coefficients of $\psi(\theta(\cdot, \cdot))$ via

$$
\begin{aligned}
& b_{d, n}= \frac{2 n+d-1}{2^{3-d} \pi} \frac{\left(\Gamma\left(\frac{d-1}{2}\right)\right)^{2}}{\Gamma(d-1)} \int_{0}^{\pi} C_{n}^{(d-1) / 2}(\cos \vartheta)(\sin \vartheta)^{d-1} \psi(\vartheta) d \vartheta \\
&= \frac{2 n+d-1}{2^{3-d} \pi} \frac{\left(\Gamma\left(\frac{d-1}{2}\right)\right)^{2}}{\Gamma(d-1)}\left(2 \pi \sigma_{d} \prod_{k=2}^{d-1} \int_{0}^{\pi}(\sin \vartheta)^{k-1} d \vartheta\right)^{-1} \\
& \quad \times \int_{\mathbb{S}^{d} \times \mathbb{S}^{d}} C_{n}^{(d-1) / 2}(\langle u, v\rangle) \psi(\theta(u, v)) d u d v \\
&=\frac{\left(\Gamma\left(\frac{d-1}{2}\right)\right)^{2} \Gamma\left(\frac{d}{2}\right)(d-1)}{\Gamma(d-1) 2^{4-d} \pi^{(d+1) / 2}}\left(\bar{c}_{d, n}\right)^{-1}\left\langle E_{d, n}, \psi(\theta(\cdot, \cdot))\right\rangle_{L^{2}} .
\end{aligned}
$$

The quotient in the previous line is positive and depends only on $d$. We denote it by $\alpha_{d}$. In particular, $\left\langle E_{d, n}, \psi(\theta(\cdot, \cdot))\right\rangle_{L^{2}} \geq 0$ for all $n \in \mathbb{N}_{0}$. We have

$$
\frac{C_{n}^{(d-1) / 2}(\langle\cdot, \cdot\rangle)}{C_{n}^{(d-1) / 2}(1)}=\sigma_{d} \bar{c}_{d, n} E_{d, n},
$$

hence

$$
\psi(\theta(\cdot, \cdot))=\alpha_{d} \sigma_{d} \sum_{n=0}^{\infty}\left\langle E_{d, n}, \psi(\theta(\cdot, \cdot))\right\rangle_{L^{2}} E_{d, n} .
$$

By Theorem 3.1

$$
\begin{aligned}
1=\sum_{n=0}^{\infty} b_{n, d}=\alpha_{d} \sum_{n=0}^{\infty}\left(\bar{c}_{d, n}\right)^{-1}\left\langle E_{d, n}, \psi(\theta(\cdot, \cdot))\right\rangle_{L^{2}} & \\
& =\alpha_{d} \sum_{n=0}^{\infty}\left(\bar{c}_{d, n}\right)^{-1}\left|\left\langle E_{d, n}, \psi(\theta(\cdot, \cdot))\right\rangle_{L^{2}}\right| ;
\end{aligned}
$$

hence Theorem 2.6 yields the claim. For $d=1$ we have

$$
b_{1, n}= \begin{cases}1 /(2 \pi)\left\langle E_{1, n}, \psi(\theta(\cdot, \cdot))\right\rangle_{L^{2}}, & \text { if } n=0, \\ \sqrt{2} /(2 \pi)\left\langle E_{1, n}, \psi(\theta(\cdot, \cdot))\right\rangle_{L^{2}}, & \text { if } n \geq 1 ;\end{cases}
$$

hence we can apply the same arguments as above. 
Remark 3.3. For a function $\psi \in \Psi_{d+k} \subset \Psi_{d}$ for some $k \geq 1$, Theorem 3.2 yields spherical convolution roots $g_{d+k} \in L_{d+k, \mathcal{I}}^{2}$ and $g_{d} \in L_{d, \mathcal{I}}^{2}$ with respect to the convolution in $\mathbb{S}^{d+k}$ and $\mathbb{S}^{d}$, respectively. The associated functions $\bar{g}_{d+k}, \bar{g}_{d}$ are both defined on $[0, \pi]$, and one would hope for a simple functional relationship between them, but it remains elusive thus far. However, on the level of Schoenberg coefficients, the functions $g_{d+2}$ and $g_{d}$ are easily put in relation using [11, Corollary 3].

Let $\psi \in \Psi_{d}$. The construction in the proofs of Theorems 2.6 and 3.2 shows that the class $\mathcal{G}_{d}(\psi)$ of all spherical convolution roots $g \in L_{d, \mathcal{I}}^{2}$ of $\psi$ is given by all functions $g \in L_{d, \mathcal{I}}^{2}$, whose Gegenbauer coefficients are given by

$$
\left(\alpha_{d}^{-\frac{1}{2}} \sigma_{n} b_{d, n}^{\frac{1}{2}}\right)_{n \in \mathbb{N}_{0}}
$$

where $\left(b_{d, n}\right)_{n \in \mathbb{N}_{0}}$ are the Schoenberg coefficients of $\psi$ and $\left(\sigma_{n}\right)_{n \in \mathbb{N}_{0}}$ is a sequence with $\sigma_{n} \in\{-1,1\}$; cf. Figure 1. In Theorem 3.2 we identify a unique convolution root by setting $\sigma_{n}=1$ for all $n \in \mathbb{N}_{0}$. This choice resolves the identifiability issue when inferring the kernel of Lévy based models for star-shaped random particles from their covariance or correlation structure as mentioned in Section 1 . See also [12,27.

We conclude the section by using the convolution representation to calculate the Schoenberg coefficients of the function

$$
\iota_{d}:[0, \pi] \rightarrow \mathbb{R}, \vartheta \mapsto \frac{1}{\nu_{d}(r)} \overline{\mathbb{1}_{\{\theta(\cdot, \cdot) \leq r\}} \circledast \mathbb{1}_{\{\theta(\cdot, \cdot) \leq r\}}}(\vartheta),
$$

where $r \in(0, \pi / 2]$ and $\nu_{d}$ is the normalizing constant ensuring that $\iota_{d}(0)=1$. Here, $\mathbb{1}_{A}$ denotes the indicator function of a set $A$. The convolution is taken in $\mathbb{S}^{d} \times \mathbb{S}^{d}$. It is a short calculation to show that $\nu_{1}(r)=2 r$. For $d \geq 2$ the normalizing constant is given by

$$
\nu_{d}(r)=\sigma_{d-1} \int_{0}^{r}(\sin \vartheta)^{d-1} d \vartheta .
$$

The function $\iota_{2}$ has been calculated explicitly in [23]. Estrade and Istas [8] provide a recursive formula for the functions $\iota_{d}, d \geq 2$.

Lemma 3.4. Let $r \in(0, \pi / 2]$. The function $\mathbb{1}_{\{\theta(\cdot, \cdot) \leq r\}} \in L_{d, \mathcal{I}}^{2}$ has Gegenbauer coefficients $\left\{\omega_{d, n}\right\}_{n \in \mathbb{N}_{0}}$ given, for $n \geq 1$, by

$$
\omega_{d, n}=c_{d, n} \sigma_{d} \sigma_{d-1} \frac{d-1}{n(n+d-1)}(\sin (r))^{d} C_{n-1}^{(d+1) / 2}(\cos (r)), \quad \text { for } d \geq 2,
$$

and $\omega_{1, n}=(2 \sqrt{2} / n) \sin (n r)$. Finally, $\omega_{d, 0}=\nu_{d}(r)$, where $\nu_{d}(r)$ is given in (3.4).

Proof. Suppose first that $d \geq 2$. We have

$$
\begin{aligned}
& \left\langle\mathbb{1}_{\{\theta(\cdot, \cdot) \leq r\}}, E_{d, n}\right\rangle_{L_{d}^{2}}=c_{d, n} \int_{\mathbb{S}^{d}} \int_{\mathbb{S}^{d}} \mathbb{1}_{\{\theta(u, v) \leq r\}} C_{n}^{(d-1) / 2}(\langle u, v\rangle) d u d v \\
& =c_{d, n} \sigma_{d}^{2}\left(\int_{0}^{\pi}(\sin \vartheta)^{d-1} d \vartheta\right)^{-1} \int_{0}^{\pi} \mathbb{1}_{\{\vartheta \leq r\}} C_{n}^{(d-1) / 2}(\cos \vartheta)(\sin \vartheta)^{d-1} d \vartheta \\
& =c_{d, n} \sigma_{d} \sigma_{d-1} \int_{\cos (r)}^{1} C_{n}^{(d-1) / 2}(u)\left(1-u^{2}\right)^{(d-2) / 2} d u
\end{aligned}
$$




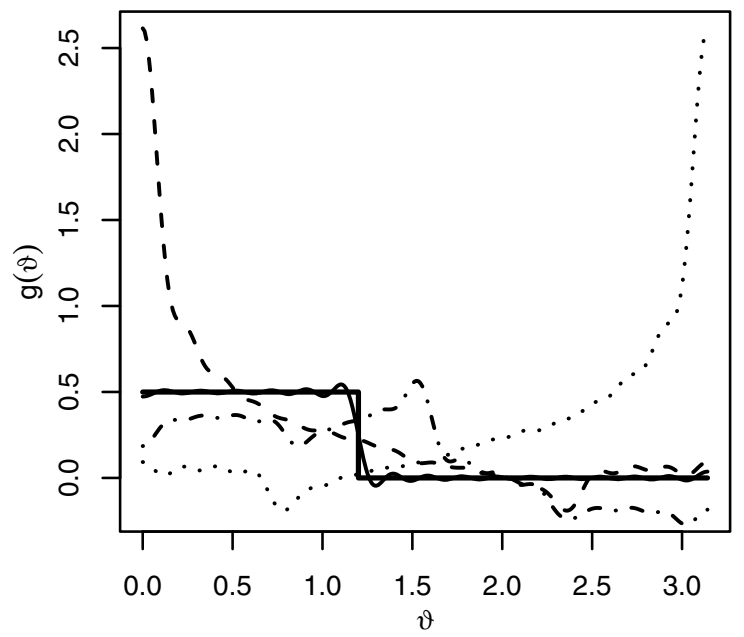

Figure 1. Different convolution roots $g$ of $\iota_{2}(r)$ for $r=1.2$. The solid lines display the function $\nu_{d}(1.2)^{-1 / 2} \mathbb{1}_{\{\vartheta \leq 1.2\}}$ and its approximation by the first 32 Gegenbauer polynomials. The dashed line is the convolution root with nonnegative Gegenbauer coefficients. The dotted line represents the convolution root with $\sigma_{n}=(-1)^{n}$, whereas the dash-dotted line has $\sigma_{n}=(-1)^{[n / 2]}$, with $\left(\sigma_{n}\right)_{n \in \mathbb{N}_{0}}$ as in (3.3).

Using $c_{d, 0}=\sigma_{d}^{-1}$, the formula for $n=0$ follows. By [6, 18.9.20] we have for $n \geq 1$

$$
\frac{d}{d x}\left(\left(1-x^{2}\right)^{d / 2} C_{n-1}^{(d+1) / 2}(x)\right)=-\frac{n(n+d-1)}{d-1}\left(1-x^{2}\right)^{(d-2) / 2} C_{n}^{(d-1) / 2}(x),
$$

which implies the lemma. The case $d=1$ is a simple calculation.

Using the relation between the Gegenbauer and the Schoenberg coefficients calculated in the proof of Theorem 3.2 we obtain the following corollary.

Corollary 3.5. The function $\iota_{d}$ is in $\Psi_{d}$. For $d \geq 2$ its Schoenberg coefficients are given by

$$
b_{d, 0}=\frac{\nu_{d}(r)}{\sigma_{d}^{2}} \frac{\Gamma\left(\frac{d-1}{2}\right)^{2} \Gamma\left(\frac{d}{2}\right)(d-1)}{\Gamma(d-1) 2^{4-d} \pi^{(d+1) / 2}},
$$

and, for $n \geq 1$,

$$
b_{d, n}=\gamma_{d}(r)(2 n+d-1) C_{n}^{(d-1) / 2}(1)\left(\frac{C_{n-1}^{(d+1) / 2}(\cos r)}{C_{n-1}^{(d+1) / 2}(1)}\right)^{2}
$$

where

$$
\gamma_{d}(r)=\frac{1}{\nu_{d}(r)} \frac{\Gamma\left(\frac{d-1}{2}\right)^{2} 2^{d-2} \pi^{(d-1) / 2}}{d^{2} \Gamma\left(\frac{d}{2}\right)}(\sin r)^{2 d} .
$$

For $d=1$, we have $b_{1,0}=r /\left(4 \pi^{3}\right)$ and $b_{1, n}=\sqrt{2} \sin ^{2}(n r) /\left(r n^{2} \pi^{2}\right)$ for $n \geq 1$. 
This example illustrates that the convolution root constructed in Theorem 3.2 may not be the most natural one. The Gegenbauer coefficients of $\nu_{d}(r)^{-1 / 2} \times$ $\mathbb{1}_{\{\theta(\cdot, \cdot) \leq r\}}$ take both positive and negative signs; cf. Lemma 3.4. Hence, it is not the convolution root of $\iota_{d}$ that results from the construction in Theorem 3.2. cf. Figure 1. The function $\iota_{d}$ is an example of a member of $\Psi_{d}$ that is supported on a spherical cap of radius $2 r$. If we would like to have a convolution root that is supported on a spherical cap of radius $r$, such as $\nu_{d}(r)^{-1 / 2} \mathbb{1}_{\{\theta(\cdot, \cdot) \leq r\}}$ for $\iota_{d}$, it may not be suitable to choose all coefficients of the convolution root nonnegative. In the Euclidean case, the existence of convolution roots with half-support, the so-called Boas-Kac roots, is discussed in [7, building on the classical result of Boas and Kac 3]. It remains an open problem whether Boas-Kac roots always exist for functions in $\Psi_{d}$.

\section{Differentiability}

4.1. Proof of Theorem 1.2. We denote by $\tilde{\Psi}_{d}$ the space of all continuous functions $\varphi:[0, \pi] \rightarrow \mathbb{R}$ which are such that the function $\varphi(\theta(\cdot, \cdot)): \mathbb{S}^{d} \times \mathbb{S}^{d} \rightarrow \mathbb{R}$ is positive definite. The difference between the spaces $\Psi_{d}$ and $\tilde{\Psi}_{d}$ is that the members $\psi \in \Psi_{d} \subset \tilde{\Psi}_{d}$ are additionally required to fulfill $\psi(0)=1$. Theorems 3.1 and 3.2 also hold for the class $\tilde{\Psi}_{d}$ with the obvious modification that we need to require $\sum_{n=0}^{\infty} b_{d, n}<\infty$ instead of $\sum_{n=0}^{\infty} b_{d, n}=1$ for the Schoenberg coefficients in the former.

For the proof of Theorem 1.2 on the differentiability of positive definite functions on spheres we show the following proposition, which can be applied iteratively to yield the assertion.

Proposition 4.1. Let $d \geq 1, \psi \in \tilde{\Psi}_{d+2}$. Then $\psi$ is continuously differentiable in $(0, \pi)$ and its derivative can be written as

$$
\psi^{\prime}(\vartheta)=\frac{1}{\sin \vartheta}\left(f_{1}(\vartheta)-f_{2}(\vartheta)\right)
$$

where $f_{1}, f_{2} \in \tilde{\Psi}_{d}$.

Proof. By [6, 18.9.19] the derivative of $C_{n}^{\alpha}$ for $\alpha>0$ and $n \geq 1$ is given by

$$
\frac{d}{d x} C_{n}^{\alpha}(x)=2 \alpha C_{n-1}^{\alpha+1}(x)
$$

We assume first that $d \geq 2$. As $\tilde{\Psi}_{d} \supset \tilde{\Psi}_{d+2}$ we can write $\psi$ as

$$
\psi(\vartheta)=\sum_{n=0}^{\infty} b_{d, n} \frac{C_{n}^{(d-1) / 2}(\cos \vartheta)}{C_{n}^{(d-1) / 2}(1)}, \quad \vartheta \in[0, \pi],
$$

with nonnegative coefficients $b_{d, n}$ such that $\sum_{n=0}^{\infty} b_{d, n}<\infty$; see Theorem 3.1. For $N \in \mathbb{N}, \vartheta \in[0, \pi]$ we define

$$
\psi_{N}(\vartheta)=\sum_{n=0}^{N} b_{d, n} \frac{C_{n}^{(d-1) / 2}(\cos \vartheta)}{C_{n}^{(d-1) / 2}(1)} .
$$


By Proposition 2.7 $\psi_{N}$ converges uniformly to $\psi$. Let $\vartheta \in(0, \pi)$. By (4.1), the derivative of $\psi_{N}$ is given by

$$
\begin{aligned}
\psi_{N}^{\prime}(\vartheta)= & \sum_{n=1}^{N} b_{d, n}(d-1) \frac{C_{n-1}^{(d+1) / 2}(\cos \vartheta)}{C_{n}^{(d-1) / 2}(1)}(-\sin \vartheta) \\
= & \frac{-1}{\sin \vartheta} \sum_{n=1}^{N} b_{d, n} \frac{1}{C_{n}^{(d-1) / 2}(1)}\left(\frac{(n+d-2)(n+d-1)}{2 n+d-1} C_{n-1}^{(d-1) / 2}(\cos \vartheta)\right. \\
& \left.\quad-\frac{n(n+1)}{2 n+d-1} C_{n+1}^{(d-1) / 2}(\cos \vartheta)\right) \\
= & \frac{1}{\sin \vartheta} \sum_{n=1}^{N} b_{d, n} \frac{n(n+d-1)}{2 n+d-1}\left(\frac{C_{n+1}^{(d-1) / 2}(\cos \vartheta)}{C_{n+1}^{(d-1) / 2}(1)}-\frac{C_{n-1}^{(d-1) / 2}(\cos \vartheta)}{C_{n-1}^{(d-1) / 2}(1)}\right),
\end{aligned}
$$

where we used (2.3) and

$$
\begin{aligned}
C_{n}^{(d+1) / 2}(\cos \vartheta)(\sin \vartheta)^{2}= & \frac{(n+d-1)(n+d)}{(d-1)(2 n+d+1)} C_{n}^{(d-1) / 2}(\cos \vartheta) \\
& -\frac{(n+1)(n+2)}{(d-1)(2 n+d+1)} C_{n+2}^{(d-1) / 2}(\cos \vartheta)
\end{aligned}
$$

see [6, equation (18.9.8)]. Therefore

$$
\begin{aligned}
(\sin \vartheta) & \psi_{N}^{\prime}(\vartheta)=-b_{d, 1} \frac{d}{d+1} \\
& +\sum_{n=0}^{N}\left(\frac{n(n+d-1)}{2 n+d-1} b_{d, n}-\frac{(n+2)(n+d+1)}{2 n+d+3} b_{d, n+2}\right) \frac{C_{n+1}^{(d-1) / 2}(\cos \vartheta)}{C_{n+1}^{(d-1) / 2}(1)} \\
& +\sum_{n=N-1}^{N} b_{d, n+2} \frac{(n+2)(n+d+1)}{2 n+d+3} \frac{C_{n+1}^{(d-1) / 2}(\cos \vartheta)}{C_{n+1}^{(d-1) / 2}(1)} .
\end{aligned}
$$

The last term in the above equation converges to zero uniformly in $\vartheta$ as $N \rightarrow$ $\infty$ by [11, Corollary 4] and Lemma 4.2. We will omit it in the sequel. Using [11, Corollary 3(b)], we obtain

$$
\begin{aligned}
& \frac{n(n+d-1)}{2 n+d-1} b_{d, n}-\frac{(n+2)(n+d+1)}{2 n+d+3} b_{d, n+2} \\
& \quad=\frac{d n}{n+d} b_{d+2, n}-\frac{d(2 n+d+1)(n+2)}{(2 n+d+3)(n+d)} b_{d, n+2} .
\end{aligned}
$$

Hence,

$$
\begin{aligned}
(\sin \vartheta) \psi_{N}^{\prime}(\vartheta)= & d \sum_{n=0}^{N} \frac{n}{n+d} b_{d+2, n} \frac{C_{n+1}^{(d-1) / 2}(\cos \vartheta)}{C_{n+1}^{(d-1) / 2}(1)} \\
& -d \sum_{n=1}^{N+2} \frac{(2 n+d-3) n}{(2 n+d-1)(n+d-2)} b_{d, n} \frac{C_{n-1}^{(d-1) / 2}(\cos \vartheta)}{C_{n-1}^{(d-1) / 2}(1)} .
\end{aligned}
$$

We set $\beta_{0}^{(1)}=0$,

$$
\beta_{n}^{(1)}=d \frac{n-1}{n+d-1} b_{d+2, n-1}, \quad \text { for } n \geq 1,
$$


and

$$
\beta_{n}^{(2)}=d \frac{(2 n+d-1)(n+1)}{(2 n+d+1)(n+d-1)} b_{d, n+1}, \quad \text { for } n \geq 0 .
$$

The sequences $\left\{\beta_{n}^{(i)}\right\}_{n \in \mathbb{N}_{0}}, i=1,2$, are nonnegative and summable by assumption. Therefore they are the Schoenberg coefficients of some functions $f_{1}, f_{2} \in \tilde{\Psi}_{d}$. By Proposition 2.7 their partial Gegenbauer sums converge uniformly, which yields the claim.

If $d=1$, the proof uses the same arguments with [11, Corollary 3(a)] instead of [11, Corollary 3(b)]. The Schoenberg coefficients of the functions $f_{1}, f_{2}$ are then given by $\beta_{n}^{(1)}=((n-1) / n) b_{3, n-1}, \beta_{n}^{(2)}=b_{1, n+1}$, for $n \geq 1$, and $\beta_{0}^{(1)}=0$, $\beta_{0}^{(2)}=(1 / 2) b_{1,1}$.

Lemma 4.2. Let $\left(\alpha_{n}\right)_{n \in \mathbb{N}}$ be an increasing sequence converging to 1 such that the sequence $\left(\alpha_{n}^{n}\right)_{n \in \mathbb{N}}$ is bounded away from 0 . Suppose that $\sum_{n=1}^{\infty} b_{n}<\infty$ for some sequence $\left(b_{n}\right)_{n \in \mathbb{N}}$ of nonnegative numbers. If

$$
b_{n} \geq \alpha_{n} b_{n+1}, \quad \text { for all } n \in \mathbb{N},
$$

then $n b_{n} \rightarrow 0$ as $n \rightarrow \infty$.

Proof. Let $\left(\alpha_{n}^{n}\right)_{n \in \mathbb{N}}$ be bounded below by $C>0$. Let $\varepsilon>0$ and choose $n_{0}$ such that $\sum_{k=n+1}^{m} b_{k}<\varepsilon$ for all $m>n>n_{0}$. With $m=2 n$ we obtain

$$
\begin{aligned}
& \varepsilon>\sum_{k=n+1}^{2 n} b_{k} \geq \sum_{k=n+1}^{2 n} \prod_{j=k}^{2 n-1} \alpha_{j} b_{2 n} \geq \sum_{k=n+1}^{2 n}\left(\alpha_{n}\right)^{2 n-k} b_{2 n} \\
& \quad \geq \alpha_{n}^{2 n} n b_{2 n} \geq C^{2} n b_{2 n} \geq 0 .
\end{aligned}
$$

Using the same argument for $m=2 n+1$ yields the claim.

4.2. Optimality of Theorem 1.2. In this section we show that Theorem 1.2 is optimal for all odd dimensions using similar ideas as in [10].

Proposition 4.3. Let $d$ be an odd integer. Then there exists a function $\psi \in \Psi_{d}$ whose derivative of order $(d-1) / 2$ is not continuously differentiable.

We are not aware of a function $\psi \in \Psi_{2}$ with discontinuous derivative. If such a function were available, our method would immediately also yield the optimality of the differentiability result in even dimensions.

For the proof of Proposition 4.3, we introduce a turning bands operator for isotropic positive definite functions on spheres in analogy to the Euclidean case, where the turning bands operator originates in the work of Matheron [14. Let $\beta=\left(\beta_{n}\right)_{n \in \mathbb{N}_{0}}$ be a sequence of real numbers. For an integer $k \in \mathbb{Z}$ we define the sequence $\beta \circ \tau_{k}$ as follows. If $k>0$ its members are

$$
\left(\beta \circ \tau_{k}\right)_{n}= \begin{cases}0, & \text { if } n<k, \\ \beta_{n-k}, & \text { if } n \geq k\end{cases}
$$

for $n \in \mathbb{N}_{0}$. If $k \leq 0$ we put $\left(\beta \circ \tau_{k}\right)_{n}=\beta_{n-k}$ for all $n \in \mathbb{N}_{0}$. Let $d \geq 1$ be an integer. For a summable sequence $\beta=\left(\beta_{n}\right)_{n \in \mathbb{N}}$ of nonnegative numbers $\beta_{n}$ we define $\psi_{d}(\beta, \vartheta)$ for $\vartheta \in[0, \pi]$ as

$$
\psi_{d}(\beta, \vartheta)=\sum_{n=0}^{\infty} \beta_{n} \frac{C_{n}^{(d-1) / 2}(\cos \vartheta)}{C_{n}^{(d-1) / 2}(1)} \in \tilde{\Psi}_{d}
$$


Proposition 4.4. Let $d \geq 1$ be an integer and let $\beta=\left(\beta_{n}\right)_{n \in \mathbb{N}}$ be a summable sequence of nonnegative numbers $\beta_{n}$. Then, for all $r \in[0, \pi]$,

$$
\psi_{d}(\beta, r)=\beta_{0}+\cos r \psi_{d+2}\left(\beta \circ \tau_{-1}, r\right)+\frac{1}{d} \sin r \psi_{d+2}^{\prime}\left(\beta \circ \tau_{-1}, r\right)
$$

and

$$
\frac{1}{d}(\sin r)^{d} \psi_{d+2}\left(\beta \circ \tau_{-1}, r\right)=\int_{0}^{r}(\sin \vartheta)^{d-1}\left(\psi_{d}(\beta, \vartheta)-\beta_{0}\right) d \vartheta
$$

Proof. Suppose first that $d \geq 2$. Using Proposition 2.7 (3.5), and (2.3) we obtain

$$
\begin{aligned}
\int_{0}^{r}(\sin \vartheta)^{d-1} \psi_{d}(\beta, \vartheta) d \vartheta & =\sum_{n=0}^{\infty} \beta_{n} \int_{0}^{r}(\sin \vartheta)^{d-1} \frac{C_{n}^{(d-1) / 2}(\cos \vartheta)}{C_{n}^{(d-1) / 2}(1)} d \vartheta \\
& =\beta_{0} \int_{0}^{r}(\sin \vartheta)^{d-1} d \vartheta+\frac{1}{d}(\sin r)^{d} \sum_{n=1}^{\infty} \beta_{n} \frac{C_{n-1}^{(d+1) / 2}(\cos r)}{C_{n-1}^{(d+1) / 2}(1)}
\end{aligned}
$$

which implies (4.3). Differentiating both sides of (4.3) with respect to $r$ yields (4.2). The case $d=1$ can be shown using the same arguments.

Lemma 4.5. Let $d \geq 1$ be an integer and let $\beta=\left(\beta_{n}\right)_{n \in \mathbb{N}}$ be a summable sequence of nonnegative numbers $\beta_{n}$. For any $k \in \mathbb{Z}$ the function $\psi_{d}\left(\beta \circ \tau_{k}, \cdot\right)$ is continuously differentiable if and only if the same holds true for $\psi_{d}(\beta, \cdot)$.

Proof. The proof of Theorem 1.2 shows that the differentiability of a function $\psi_{d}(\beta, \cdot)$ depends only on the nonnegativity and asymptotic properties of the sequence $\left(\beta_{n}\right)_{n \in \mathbb{N}_{0}}$.

Proof of Proposition 4.3. Let $c \in(0, \pi)$. Then the function

$$
\psi(\vartheta)=\max \left\{0,\left(1-\frac{\vartheta}{c}\right)\right\}, \quad \vartheta \in[0, \pi]
$$

belongs to the class $\Psi_{1}$ as can be shown by elementary arguments. Its first derivative does not exist at the point $\vartheta=c$. Let $\beta=\left(\beta_{n}\right)_{n \in \mathbb{N}_{0}}$ be the sequence of 1-dimensional Schoenberg coefficients of $\psi$. Let $d \geq 3$ be an odd integer. By (4.3) and Lemma 4.5. the function $\psi_{d}\left(\beta \circ \tau_{-(d-1) / 2}, \vartheta\right) \in \Psi_{d}$ and its derivative of order $1+(d-1) / 2$ do not exist at $\vartheta=c$.

The truncated power functions $\psi(\vartheta)=\max \left\{0,(1-\vartheta / c)^{\tau}\right\}$ were studied in detail in [2. The authors were able to show that they belong to $\Psi_{d}$ if $\tau \geq(d+1) / 2$ for $d=3,5,7$ and conjectured the result for all dimensions. Theorem 1.2 immediately shows the necessity of the condition for all odd dimensions.

\section{ACKNOWLEDGEMENT}

The author would like to thank Tilmann Gneiting for interesting and encouraging discussions. 


\section{REFERENCES}

[1] Sudipto Banerjee, On geodetic distance computations in spatial modeling, Biometrics 61 (2005), no. 2, 617-625, DOI 10.1111/j.1541-0420.2005.00320.x. MR2140936

[2] R. K. Beatson, W. zu Castell, and Y. Xu, A Pólya criterion for (strict) positive definiteness on the sphere, IMA J. Numer. Anal. (2013), DOI 10.1093/imanum/drt008.

[3] R. P. Boas Jr. and M. Kac, Inequalities for Fourier transforms of positive functions, Duke Math. J. 12 (1945), 189-206. MR0012152(6,265h)

[4] Roberto Cavoretto and Alessandra De Rossi, Fast and accurate interpolation of large scattered data sets on the sphere, J. Comput. Appl. Math. 234 (2010), no. 5, 1505-1521, DOI 10.1016/j.cam.2010.02.031. MR.2610367 (2011c:65021)

[5] Debao Chen, Valdir A. Menegatto, and Xingping Sun, A necessary and sufficient condition for strictly positive definite functions on spheres, Proc. Amer. Math. Soc. 131 (2003), no. 9, 2733-2740 (electronic), DOI 10.1090/S0002-9939-03-06730-3. MR1974330 (2004d:43007)

[6] Digital Library of Mathematical Functions, Release date 2012-03-23, National Institute of Standards and Technology from http://dlmf.nist.gov/, 2011.

[7] Werner Ehm, Tilmann Gneiting, and Donald Richards, Convolution roots of radial positive definite functions with compact support, Trans. Amer. Math. Soc. 356 (2004), no. 11, 46554685 (electronic), DOI 10.1090/S0002-9947-04-03502-0. MR2067138(2005g:42012)

[8] Anne Estrade and Jacques Istas, Ball throwing on spheres, Bernoulli 16 (2010), no. 4, 953970, DOI 10.3150/09-BEJ241. MR2759164 (2011m:60029)

[9] Gregory E. Fasshauer and Larry L. Schumaker, Scattered data fitting on the sphere, Mathematical methods for curves and surfaces, II (Lillehammer, 1997), Innov. Appl. Math., Vanderbilt Univ. Press, Nashville, TN, 1998, pp. 117-166. MR1640548 (99d:41054)

[10] Tilmann Gneiting, On the derivatives of radial positive definite functions, J. Math. Anal. Appl. 236 (1999), no. 1, 86-93, DOI 10.1006/jmaa.1999.6434. MR.1702687 (2000k:42012)

[11] Tilmann Gneiting, Strictly and non-strictly positive definite functions on spheres, Bernoulli 19 (2013), no. 4, 1327-1349. MR3102554

[12] L. V. Hansen, T. L. Thorarinsdottir, and T. Gneiting, Lévy particles: Modelling and simulating star-shaped random sets, CSGB Research Report (2011).

[13] Chunfeng Huang, Haimeng Zhang, and Scott M. Robeson, On the validity of commonly used covariance and variogram functions on the sphere, Math. Geosci. 43 (2011), no. 6, 721-733, DOI 10.1007/s11004-011-9344-7. MR2824128(2012g:86019)

[14] G. Matheron, Quelque Aspects de la Montée, Note Géostatistique 120, Centre de Géostatistique, Fontainebleau, France, 1972.

[15] Valdir A. Menegatto, Strictly positive definite kernels on the Hilbert sphere, Appl. Anal. 55 (1994), no. 1-2, 91-101, DOI 10.1080/00036819408840292. MR1379646 (96m:41054)

[16] V. A. Menegatto, C. P. Oliveira, and A. P. Peron, Strictly positive definite kernels on subsets of the complex plane, Comput. Math. Appl. 51 (2006), no. 8, 1233-1250, DOI 10.1016/j.camwa.2006.04.006. MR2235825(2007c:41021)

[17] Allan Pinkus, Strictly Hermitian positive definite functions, J. Anal. Math. 94 (2004), 293318, DOI 10.1007/BF02789051. MR2124464 (2005k:43004)

[18] M. Reimer, Multivariate Polynomial Approximations, Birkhäuser Verlag, Basel, 2003. MR2003508 (2004i:41001)

[19] I. J. Schoenberg, Metric spaces and completely monotone functions, Ann. of Math. (2) 39 (1938), no. 4, 811-841, DOI 10.2307/1968466. MR1503439

[20] I. J. Schoenberg, Positive definite functions on spheres, Duke Math. J. 9 (1942), 96-108. $\operatorname{MR} 0005922(3,232 \mathrm{c})$

[21] Michael Schreiner, Locally supported kernels for spherical spline interpolation, J. Approx. Theory 89 (1997), no. 2, 172-194, DOI 10.1006/jath.1997.3037. MR1447837(98j:41017)

[22] S. Soubeyrand, J. Enjalbert, and I. Sache, Accounting for roughness of circular processes: Using Gaussian random processes to model the anisotropic spread of airborne plant disease, Theor. Popul. Biol. 73 (2008), 92-103.

[23] A. Tovchigrechko and I. A. Vakser, How common is the funnel-like energy landscape in protein-protein interactions?, Protein Science 10 (2001), 1572-1583.

[24] Dirk Werner, Funktionalanalysis (German, with German summary), Third, revised and extended edition, Springer-Verlag, Berlin, 2000. MR1787146 (2001i:46001) 
[25] Andrew T. A. Wood, When is a truncated covariance function on the line a covariance function on the circle?, Statist. Probab. Lett. 24 (1995), no. 2, 157-164, DOI 10.1016/01677152(94)00162-2. MR.1354370 (97b:60061)

[26] Yuan Xu and E. W. Cheney, Strictly positive definite functions on spheres, Proc. Amer. Math. Soc. 116 (1992), no. 4, 977-981, DOI 10.2307/2159477. MR1096214 (93b:43005)

[27] J. Ziegel, Stereological modelling of random particles, Comm. Statist. Theory Methods 42 (2013), no. 7, 1428-1442. MR3031290

Department of Mathematics and Statistics, University of Bern, Institute of Mathematical Statistics and Actuarial Science, Sidlerstrasse 5, 3012 Bern, Switzerland

E-mail address: johanna.ziegel@stat.unibe.ch 